\section{Parto acidental não-hospitalar como indicador de risco para a mortalidade infantil}

\section{Accidental non-hospital birth as an indicator of risk of infant mortality}

Zilda Pereira da Silva 1

Márcia Furquim de Almeida 2

Gizelton Pereira Alencar 3

1-3 Departamento de Epidemiologia. Faculdade de Saúde Pública. Universidade de São Paulo. Av. Dr. Arnaldo, 715. Cerqueira César. São Paulo, SP, Brasil. CEP: 01246-904. E-mail: zildapereira@usp.br

\begin{abstract}
Objectives: to examine diferences in infant mortality rates by place of birth, in the State of São Paulo (2009).

Methods: a cohort of all 252,201 live vaginal births, with 3,289 infant deaths, was obtained from a deterministic linkage and divided into those born in hospital $(250,850)$ and those born at home or elsewhere (1351). The probability of death and relative risk $(R R)$ were calculated and a multinomial logistic regression model was used to assess the effect of covariables on mortality.

Results: $0.5 \%$ live births occurred in the home or elsewhere outside the hospital and presented a greater likelihood of mortality $(45.2$ per thousand live births) compared with those born in hospital (12.9). Mortality was higher for births outside of hospital for all types of infant mortality: early neonatal $(R R=3.9)$, late neonatal $(R R=2.6)$ and post-neonatal $(R R=3.4)$. The likelihood of death diminished as birth weight increased, although the risk of death for live births $\geq 2500 \mathrm{~g}$ in the home or elsewhere was twice as high as for hospital births. After adjustment, being born outside of hospital continued to be a risk factor for post-neonatal mortality.

Conclusions: although few in number, births outside of hospital present a greater risk of death, including post-neonatal mortality, suggesting that there are barriers to access not only during the prenatal period and delivery, but also throughout the first year of life.
\end{abstract}

Key words Infant mortality, Home childbirth, Health information system

\section{Resumo}

Objetivos: analisar diferenças na mortalidade infantil, segundo local do parto, no Estado de São Paulo (2009).

Métodos: coorte de 252.201 nascidos vivos (NV) por parto vaginal, vinculados a 3289 óbitos infantis, por técnica determinística, divididos em: nascidos em hospitais (250.850) e em domicílio/outro local (1351). Foram calculadas probabilidades de morte e os riscos relativos ( $R R$ ) e para avaliar o efeito de covariáveis sobre o óbito, utilizou-se modelo de regressão logística multinomial.

Resultados: $0,5 \% \quad \mathrm{NV}$ ocorreram em domicílio/outro local e apresentaram maior probabilidade de morte (45,2 por mil NV) do que os nascidos em hospitais (12,9). A mortalidade foi maior para os nascimentos fora do hospital em todos os componentes da mortalidade infantil: neonatal precoce $(R R=3,9)$, neonatal tardio $(R R=2,6)$ e pós-neonatal $(R R=3,4)$. A probabilidade de morte diminuiu conforme aumentou o peso ao nascer, porém o risco de morte dos $N V \geq 2500 \mathrm{~g}$ em domicílio/outro local foi duas vezes maior que nos partos hospitalares. Após ajuste, nascer fora do hospital permaneceu como risco apenas para a mortalidade pós-neonatal.

Conclusões: embora reduzidos, os partos fora do hospital apresentam maior risco de morte, inclusive no periodo pós-neonatal, sugerindo que há barreiras de acesso não só durante o pré-natal e parto, mas que estas persistem na atenção à criança no primeiro ano de vida.

Palavras-chave Mortalidade infantil, Parto domiciliar, Sistemas de informação em saúde 


\section{Introdução}

A organização da atenção ao parto varia muito entre as nações. Em alguns países, trata-se de um evento quase que totalmente hospitalar, 1,2 enquanto em outros existem opções de programas de atenção ao parto domiciliar e em centros de nascimento (birth centers), com atendimento realizado por enfermeiras-obstetrizes. 1,3-5 No Brasil, a frequência de partos hospitalares aumentou rapidamente durante o século XX. De predominante no início do século, o parto domiciliar caiu para $16,6 \%$, na década de $1980^{6}$ e atingiu $0,9 \%$, em 2011.7 Nascer em casa tornou-se, assim, um fenômeno circunscrito a áreas com maior presença de população rural e a localidades da Região Norte. ${ }^{8}$ Em áreas mais urbanizadas, como o Estado de São Paulo, desde o final dos anos 1990 os partos institucionais superam $99 \%$, sendo o parto domiciliar majoritariamente acidental.

No entanto, ainda que os partos em domicílio respondam por parcela cada vez menor dos nascimentos, estudos apontam características sociais e epidemiológicas específicas desse grupo, além de associação com resultados adversos, quando comparados tanto aos partos domiciliares planejados como aos hospitalares.9,10-13 A literatura identifica alguns fatores de natureza socioeconômica associados ao parto domiciliar acidental ou não planejado: maior frequência de mães sem companheiro, 9,11 desemprego, 12 baixa escolaridade6,11 e elevada paridade. ${ }^{9}$ Entre as características do recém-nascido, há maior prevalência de baixo peso ao nascer e de gestação pré-termo.9,10,11

Em países com programas de assistência ao parto em domicílio, investigam-se características e segurança dos nascimentos planejados para ocorrer em casa confrontando-os aos que acontecem em birth centers e/ou hospitais.3,4,5,10 Nos países que não contam com esse tipo de programa, também há preocupação em compreender as características e desfechos dos partos ocorridos acidentalmente no domicílio. 3,4,10,14 Algumas dessas pesquisas contemplam o estudo de variáveis relativas ao acesso aos serviços de atenção pré-natal e ao parto, encontrando maior frequência de assistência pré-natal ausente ou insuficiente e maior distância e/ou maior tempo de viagem da casa até a maternidade. $8,11,14$ Outras ainda apontam maior proporção de desfechos negativos para o nascimento em parto domiciliar acidental, incluindo maior probabilidade de morte. $10,13,15,16$

Assim, este estudo teve por objetivo analisar as diferenças nas características dos nascidos vivos e da mortalidade infantil, segundo local de nascimento, no Estado de São Paulo, em 2009.

\section{Métodos}

Estudo baseado em dados secundários de uma coorte de nascidos vivos (NV) vinculados aos respectivos óbitos infantis, por técnica determinística aplicada pela Fundação Sistema Estadual de Análise de Dados (Seade). A população de estudo compreende os nascidos vivos no Estado de São Paulo, no período de 01/01/2009 a 31/12/2009, e os óbitos de menores de um ano pertencentes a esta coorte, que compreendem aqueles ocorridos entre 01/01/2009 a 30/12/2010.

Foi utilizada a base unificada de eventos vitais do Estado de São Paulo, gerada a partir de dados coletados pela Fundação Seade nos cartórios do registro civil e dos dados das secretarias municipais recebidos pela Secretaria de Estado da Saúde, que alimentam o Sistema de Informações sobre Nascidos Vivos (Sinasc) e o Sistema de Informações sobre Mortalidade (SIM).

O processo de vinculação dos dados foi executado pela Fundação Seade, que utilizou metodologia de trabalho baseada em técnica determinística.17 A linkage consistiu na vinculação das bases de dados de nascidos vivos e de óbitos infantis. A rotina de vinculação estabelecida pela Fundação Seade contempla quatro etapas: a primeira consiste na seleção e padronização de variáveis e geração de variáveis derivadas; a segunda define critérios de vinculação; a terceira procede à formação de pares por igualdade ou semelhança; a quarta refere-se à busca nominal nas bases de dados e documentos originais. As variáveis selecionadas foram: nome, iniciais do nome, primeiro nome, nome da mãe, primeiro nome da mãe, iniciais do nome da mãe, data de nascimento, número da declaração de nascido vivo (DN) e declaração de óbito (DO), endereço de residência e município de residência.

O emprego dessa base de dados possibilitou a obtenção do nome da criança, o que nem sempre é viável quando se utilizam apenas as declarações de nascido vivo (DN) e de óbito (DO). Como para realizar o registro do nascimento e do óbito junto aos cartórios de registro civil é necessária a identificação nominal das pessoas, foi possível adotar o nome da criança como variável primária do processo de linkage.

Foram estudados os nascidos vivos de mães residentes e ocorridos no Estado e os respectivos óbitos infantis. Os critérios de exclusão foram recémnascido com local de parto ignorado, peso ao nascer ignorado ou menor de $500 \mathrm{~g}$ e óbito por anencefalia, dada a probabilidade de morte praticamente igual a 1 , independentemente do local de nascimento. Da 
base original (597.944 NV), inicialmente foram excluídos 3544 registros, sendo 3014 sobreviventes e 530 óbitos, que atendiam os critérios de exclusão. Após as exclusões foram selecionados apenas os nascidos vivos por parto normal.

Assim, foram estudados 252.201 nascidos vivos por parto vaginal e 3289 óbitos de menores de um ano, divididos em dois grupos: os que nasceram em estabelecimentos de saúde (250.850) e os que nasceram em domicílio ou outro lugar (1351). Destes, $78 \%$ nasceram em domicílio e $22 \%$, em geral, a caminho do hospital, na ambulância, táxi ou carro particular. O local do nascimento (estabelecimento de saúde, domicílio ou outro local) foi identificado a partir das informações da DN.

Embora o Sinasc não disponha de informações sobre a condição de planejamento ou não do parto domiciliar, pode-se dizer que no Estado de São Paulo esse fenômeno é predominantemente acidental, uma vez que não há programas governamentais que dêem suporte à realização de partos domiciliares.

Foram estudadas as características dos recémnascidos nos dois grupos, a partir das variáveis relativas à mãe (idade, escolaridade e estado civil), do recém-nascido (idade gestacional e peso ao nascer) e de realização de consultas de pré-natal. A variável paridade não foi utilizada em razão da elevada incompletude. As proporções foram comparadas tendo os nascimentos em estabelecimentos de saúde como referência, as diferenças foram avaliadas pelo teste qui-quadrado.

Para analisar a mortalidade infantil e seus componentes (neonatal precoce - 0 a 6 dias; neonatal tardio - 7 a 27 dias; e pós-neonatal - 28 a 364 dias), foram calculadas as probabilidades de morte, os riscos relativos (RR) e respectivos intervalos de confiança (IC95\%), tomando-se a probabilidade de morte dos nascidos vivos em estabelecimentos de saúde/hospitais como referência $(\mathrm{RR}=1)$ na comparação com os demais locais de nascimento. Para avaliar o efeito de covariáveis sobre o óbito infantil por componentes, foi utilizado o modelo de regressão logística multinomial. A qualidade do ajuste do modelo foi avaliado por meio do teste de Hosmer e Lemeshow para modelos de regressão logística multinomial.18 Adotou-se nível de significância de 0,05 para os testes estatísticos. Os dados foram processados utilizando-se o software Statistical Package for the Social Sciences - SPSS versão 15.0 .

Este estudo utilizou dados secundários e foi aprovado pelo Comitê de Ética da Faculdade de Saúde Pública da Universidade de São Paulo $\left(n^{\circ}\right.$ 232.359 e CAEE no 12335713.2.0000.5421).

\section{Resultados}

Os partos normais (252.201) responderam por $42 \%$ dos nascidos vivos no Estado de São Paulo. Desses, $99,5 \%$ ocorreram em estabelecimentos de saúde e $0,5 \%$ fora de hospital, em domicílio (1055) ou outro local (296), em geral no transporte a caminho do hospital ou ainda em via pública, representando respectivamente, $0,42 \%$ e $0,18 \%$ do total de partos. Em relação à residência, $57,4 \%$ das mães residiam na Região Metropolitana de São Paulo (RMSP), proporção que aumenta para $62,8 \%$ para aquelas cujo parto ocorreu fora do hospital.

Os dois grupos apresentaram características distintas e estatisticamente significantes em relação ao perfil das mães e dos recém-nascidos, bem como da realização do pré-natal. Os nascidos fora do ambiente hospitalar exibem menor proporção de mães adolescentes (11,5\%) e maior de mães com 35 anos e mais $(12,7 \%)$ do que os que nasceram em hospitais (21,6\% e $8,8 \%$, respectivamente). As mães que tiveram parto domiciliar ou em outro local apresentam menor escolaridade $(44,2 \%$ com menos de 8 anos de estudo) e maior proporção sem companheiro (77,6\%) (Tabela 1).

Os nascidos vivos em domicílio/outro local apresentaram maior proporção de pré-termos $(14,5 \%) \mathrm{e}$ de baixo peso ao nascer $(22,4 \%)$. A proporção de $\mathrm{RN}$ prematuro foi o dobro entre os que nasceram no domicílio/outro local do que os que nasceram no hospital.

O número de consultas de pré-natal também foi distinto, 20,4\% das mães que pariram em domicílio/outro local não realizaram pré-natal e apenas $31,0 \%$ fizeram sete ou mais consultas, enquanto que para as mães que deram à luz na rede hospitalar essa proporção atingiu $67,4 \%$.

Os nascidos vivos em domicílio/outro local apresentaram maior probabilidade de morte $(45,15$ por mil nascidos vivos) do que aqueles que nasceram em estabelecimentos de saúde (12,87\%o NV), com RR de 3,5 (IC95\%: 2,74 - 4,50), ou seja, o risco de morrer antes de completar um ano foi 3,5 vezes maior para quem nasceu fora do hospital (Tabela 2).

A probabilidade de morte foi maior no período neonatal precoce $(6,53$ por mil nascidos vivos), do que no neonatal tardio $(2,58 \%$ o NV) e no pósneonatal $(3,93 \%$ o NV). A comparação segundo local de nascimento mostrou que a probabilidade de morte foi maior para os nascimentos fora do ambiente hospitalar, em todos os componentes da mortalidade infantil: no período neonatal precoce com $\mathrm{RR}=3,9$ (IC95\%: 2,80 - 5,47), no neonatal tardio com $\mathrm{RR}=$ 2,6 (IC95\%: 1,35 - 5,02) e no pós-neonatal com 
Tabela 1

Nascidos vivos segundo características da mãe, da gestação e dos recém-nascidos, por local de nascimento. Estado de São Paulo, Brasil, 2009.

\begin{tabular}{|c|c|c|c|}
\hline Características & Hospital & Domicílio/outro local & $p^{*}$ \\
\hline \multicolumn{4}{|l|}{ Idade da mãe (anos) } \\
\hline Menos de 20 & 21,6 & 11,5 & $<0,001$ \\
\hline 20 a 34 & 69,6 & 75,7 & \\
\hline 35 ou mais & 8,8 & 12,7 & \\
\hline \multicolumn{4}{|l|}{ Escolaridade (anos) } \\
\hline Menos de 8 & 29,4 & 44,2 & $<0,001$ \\
\hline 8 ou mais & 69,8 & 51,0 & \\
\hline \multicolumn{4}{|l|}{ Estado civil } \\
\hline Com companheiro & 29,7 & 18,6 & $<0,001$ \\
\hline Sem companheiro & 69,0 & 77,6 & \\
\hline \multicolumn{4}{|c|}{ Idade gestacional (semanas) } \\
\hline Menos de 36 & 7,8 & 14,5 & $<0,001$ \\
\hline 37 ou mais & 91,7 & 77,9 & \\
\hline \multicolumn{4}{|l|}{ Peso ao nascer (g) } \\
\hline Menos de 2500 & 8,7 & 22,4 & $<0,001$ \\
\hline Menos de 1500 & 1,2 & 6,2 & \\
\hline De 1500 a 2499 & 7,5 & 16,2 & \\
\hline 2500 ou mais & 91,3 & 77,6 & \\
\hline \multicolumn{4}{|l|}{ Consultas de pré-natal } \\
\hline Nenhuma & 1,8 & 20,4 & $<0,001$ \\
\hline 1 a 3 & 5,8 & 14,6 & \\
\hline 4 a 6 & 24,3 & 27,6 & \\
\hline 7 ou mais & 67,4 & 31,0 & \\
\hline
\end{tabular}

Fonte dos dados brutos: Fundação Seade. Nota: exclui os ignorados; * Teste qui-quadrado.

$\mathrm{RR}=3,4$ (IC95\%: 2,16 - 5,46) (Tabela 2).

Conforme aumentou o peso ao nascer, diminuiu a probabilidade de morte, sendo $102,49 \%$ o NV para os recém-nascidos com $<2500 \mathrm{~g}$ e $4,40 \%$ o NV para os com $2500 \mathrm{~g}$ e mais. Essa tendência foi observada nos dois grupos (hospital e domicílio/outro local), sendo que, para os que nasceram fora do hospital, o risco de morrer foi mais elevado do que entre os que nasceram em hospitais. A diferença por local do parto se manteve nos dois grupos de peso, porém entre os NV com peso adequado, o risco de morte foi duas vezes maior para os que nasceram fora do hospital (Tabela 3).

A Tabela 4 mostra o modelo de regressão logística multinomial que avaliou o efeito de covariáveis sobre o óbito infantil por idade ao morrer. Observouse que, após o ajuste pelas demais variáveis, nascer fora do hospital mostrou-se como risco apenas para a mortalidade pós-neonatal. A ausência ou pequeno número de consultas de pré-natal, nascimentos de baixo peso e pré-termo mostraram-se associados à mortalidade neonatal precoce e tardia e pósneonatal. A escolaridade materna não estava associada aos óbitos neonatais tardios e mostrou ter um efeito protetor para os óbitos neonatais precoces, porém como pode ser observado na tabela, o limite superior do intervalo de confiança é muito próximo de 1. Verificou-se também que os diferenciais de mortalidade neonatal precoce segundo escolaridade são muito pequenos (dados não apresentados). Com exceção da idade materna, todas as variáveis de estudo estavam associadas à ocorrência de óbitos no período pós-neonatal. O teste de Hosmer e Lemeshow indicou boa qualidade do ajuste do modelo $(p<0,002)$. 
Nascidos vivos, óbitos, probabilidade de morte e risco relativo (RR), segundo idade ao morrer e local de nascimento. Estado de São Paulo, Brasil, 2009.

\begin{tabular}{|c|c|c|c|c|c|c|c|}
\hline $\begin{array}{l}\text { Idade ao morrer e local } \\
\text { de nascimento }\end{array}$ & $\begin{array}{c}\text { Nascidos } \\
\text { vivos }\end{array}$ & Óbitos & Sobreviventes & $\begin{array}{l}\text { Probabilidade de } \\
\text { morte (1) }\end{array}$ & RR & IC95\% & $p^{*}$ \\
\hline$<1$ ano & & & & & & & $<0,001$ \\
\hline Hospital & 250.850 & 3.228 & 247.622 & 12,87 & 1 & & \\
\hline Domicílio/outro local & 1.351 & 61 & 1.290 & 45,15 & 3,50 & $2,74-4,50$ & \\
\hline Total & 252.201 & 3.289 & 248.912 & 13,04 & & & \\
\hline 0 a 6 dias & & & & & & & $<0,001$ \\
\hline Hospital & 250.850 & 1.614 & 249.236 & 6,43 & 1 & & \\
\hline Domicílio/outro local & 1.351 & 34 & 1.317 & 25,17 & 3,91 & $2,80-5,47$ & \\
\hline Sub-total & 252.201 & 1.648 & 250.553 & 6,53 & & & \\
\hline 7 a 27 dias & & & & & & & 0,003 \\
\hline Hospital & 250.850 & 641 & 250.209 & 2,56 & 1 & & \\
\hline Domicílio/outro local & 1.351 & 9 & 1.342 & 6,66 & 2,61 & $1,35-5,02$ & \\
\hline Sub-total & 252.201 & 650 & 251.551 & 2,58 & & & \\
\hline 28 a 364 dias & & & & & & & $<0,001$ \\
\hline Hospital & 250.850 & 972 & 249.878 & 3,87 & 1 & & \\
\hline Domicílio/outro local & 1.351 & 18 & 1.333 & 13,32 & 3,44 & $2,16-5,46$ & \\
\hline Sub-total & 252.201 & 990 & 251.211 & 3,93 & & & \\
\hline
\end{tabular}

Fonte dos dados brutos: Fundação Seade; (1) Por mil nascidos vivos; * Teste qui-quadrado.

Tabela 3

Nascidos vivos, óbitos, probabilidade de morte e risco relativo (RR), segundo peso ao nascer e local de nascimento. Estado de São Paulo, Brasil, 2009.

\begin{tabular}{|c|c|c|c|c|c|c|c|}
\hline $\begin{array}{l}\text { Peso ao nascer e local } \\
\text { de nascimento }\end{array}$ & $\begin{array}{c}\text { Nascidos } \\
\text { vivos }\end{array}$ & Óbitos & Sobreviventes & $\begin{array}{l}\text { Probabilidade de } \\
\text { morte (1) }\end{array}$ & $\mathbf{R R}$ & IC95\% & $p^{*}$ \\
\hline Menos de $2500 \mathrm{~g}$ & & & & & & & $<0,001$ \\
\hline Hospital & 21.924 & 2.227 & 19.697 & 101,58 & 1 & & \\
\hline Domicílio/outro local & 303 & 51 & 252 & 168,32 & 1,66 & $1,29-2,14$ & \\
\hline Total & 22.227 & 2.278 & 19.949 & 102,49 & & & \\
\hline $2500 \mathrm{~g}$ ou mais & & & & & & & 0,012 \\
\hline Hospital & 228.926 & 1.001 & 227.925 & 4,37 & 1 & & \\
\hline Domicílio/outro local & 1.048 & 10 & 1.038 & 9,54 & 2,18 & $1,17-4,06$ & \\
\hline Total & 229.974 & 1.011 & 228.963 & 4,40 & & & \\
\hline
\end{tabular}

Fonte dos dados brutos: Fundação Seade; (1) Por mil nascidos vivos; * Teste qui-quadrado. 
Modelo multinomial logístico para mortalidade infantil, segundo idade ao morrer, São Paulo, 2009.

\begin{tabular}{|c|c|c|c|c|c|c|c|c|c|}
\hline \multirow[t]{3}{*}{ Variáveis } & \multicolumn{9}{|c|}{ Idade ao morrer } \\
\hline & \multicolumn{3}{|c|}{0 a 6 dias } & \multicolumn{3}{|c|}{7 a 27 dias } & \multicolumn{3}{|c|}{28 a 364 dias } \\
\hline & RR & IC95\% & $p$ & $\mathrm{RR}$ & IC95\% & $p$ & $\mathrm{RR}$ & IC95\% & $p$ \\
\hline Hospital & 1 & & & 1 & & & 1 & & \\
\hline Domicílio/outro local & 1,1 & $0,72-1,69$ & 0,662 & 0,84 & $0,39-1,81$ & 0,661 & 1,76 & $1,08-2,86$ & 0,023 \\
\hline \multicolumn{10}{|l|}{ Idade mãe (anos) } \\
\hline Menos de 20 & 1,03 & $0,92-1,16$ & 0,663 & 0,99 & $0,81-1,19$ & 0,878 & 1,11 & $0,96-1,29$ & 0,160 \\
\hline De 20 a 34 & 1 & & & 1 & & & 1 & & \\
\hline 35 ou mais & 0,93 & $0,77-1,13$ & 0,476 & 1,18 & $0,91-1,54$ & 0,219 & 1,06 & $0,85-1,32$ & 0,601 \\
\hline Menos de 8 & 0,88 & $0,78-0,99$ & 0,032 & 1,00 & $0,84-1,19$ & 0,970 & 1,43 & $1,26-1,63$ & $<0,001$ \\
\hline 8 ou mais & 1 & & & 1 & & & 1 & & \\
\hline \multicolumn{10}{|l|}{ Consulta pré-natal } \\
\hline Nenhuma & 4,36 & $3,58-5,29$ & 0,000 & 3,15 & $2,30-4,32$ & 0,000 & 3,33 & $2,60-4,28$ & $<0,001$ \\
\hline 0 a 3 & 3,57 & $3,07-4,15$ & 0,000 & 2,68 & $2,12-3,40$ & 0,000 & 2,02 & $1,65-2,47$ & $<0,001$ \\
\hline 4 a 6 & 1,75 & $1,53-2,00$ & 0,000 & 1,72 & $1,41-2,09$ & 0,000 & 1,34 & $1,15-1,56$ & $<0,001$ \\
\hline 7 ou mais & 1 & & & 1 & & & 1 & & \\
\hline \multicolumn{10}{|c|}{ Idade gestacional (semanas) } \\
\hline Menos de 37 & 9,27 & $7,88-10,90$ & 0,000 & 6,53 & $5,18-8,23$ & 0,000 & 2,56 & $2,14-3,06$ & $<0,001$ \\
\hline 37 ou mais & 1 & & & 1 & & & 1 & & \\
\hline \multicolumn{10}{|l|}{ Peso ao nascer (g) } \\
\hline Menos de 2500 & 10,53 & $8,90-12,46$ & 0,000 & 9,23 & $7,25-11,75$ & 0,000 & 3,99 & $3,36-4,75$ & $<0,001$ \\
\hline 2500 ou mais & 1 & & & 1 & & & 1 & & \\
\hline
\end{tabular}

\section{Discussão}

A institucionalização do parto no Estado é bastante elevada, constatada pela baixa presença de partos normais em domicílio ou outro local, atingindo assim proporção semelhante à de países desenvolvidos, como Estados Unidos, ${ }^{2}$ Bélgica1 e França. ${ }^{14}$ A maioria dos partos ocorridos fora do hospital aconteceu no domicílio da gestante, com pequena parcela ocorrendo no caminho para o hospital, na ambulância, no táxi ou carro particular.

Como no Sinasc não é possível distinguir se o parto domiciliar foi planejado ou não, todos foram considerados acidentais, ou seja, não planejados. Estudo realizado no município de São Paulo, em 2000/2001, identificou que todos os partos ocorridos em domicílio foram acidentais. ${ }^{11}$ Porém, nos últimos anos, o parto realizado em casa com o suporte de parteiras e obstetrizes aparece como fenômeno emergente em áreas urbanas do país, mas ainda de pequena magnitude. Dados da Secretaria Municipal de Saúde (SMS) de São Paulo indicam que 16,5\%
(95) dos partos domiciliares foram assistidos por enfermeiros e médicos, em 2010.19

Em relação às características sociodemográficas, as mães que tiveram partos domiciliares/outro local apresentaram perfil mais adverso do que as atendidas nos hospitais. Essa situação de maior vulnerabilidade favorece a ocorrência de desfechos negativos em relação ao nascimento (prematuridade e baixo peso), que estão no caminho causal da mortalidade, tanto no período neonatal como no pós-neonatal.20,21

A maior proporção de mães com mais de 34 anos, baixa escolaridade e sem companheiro entre os nascidos vivos fora do ambiente hospitalar condiz com outros achados. Almeida et al. ${ }^{11}$ também observaram o mesmo em relação à escolaridade e ao estado conjugal, em estudo baseado em entrevistas domiciliares sobre mortalidade perinatal na região sul do Município de São Paulo. Na Finlândia também foram identificadas maiores prevalências de mães solteiras entre os nascimentos acidentais ocorridos fora da rede hospitalar. 9 A idade mais elevada das mães que pariram fora do hospital corrobora 
estudos prévios na Finlândia ${ }^{9}$ e França. ${ }^{22}$ Nesses lugares também foi observado maior frequência de mães multíparas, cuja progressão rápida do trabalho de parto pode aumentar o risco de parto acidental não-hospitalar.

Entre as variáveis socioeconômicas, a educação materna tem sido uma das mais estudadas em razão de seu papel fundamental na melhoria das condições de saúde maternas e na redução consistente da mortalidade infantil, devido à maior percepção, por parte das mães, dos problemas de saúde, da importância dos cuidados com o recém-nascido, da adoção de estilo de vida saudáveis e do maior acesso aos serviços básicos de saúde. ${ }^{21}$

Em estudos realizados na França14 e na Finlândia, ${ }^{9}$ outras variáveis que indicam vulnerabilidade social, tais como desemprego materno, ocupação não qualificada e residir em área rural, também estiveram associados ao parto acidental nãohospitalar; essas condições podem refletir dificuldades no uso dos serviços de saúde durante a gravidez e no parto. Porém, tais variáveis não puderam ser investigadas no presente estudo, pois não constam no Sinasc.

Identificou-se que a ausência de pré-natal foi 11 vezes mais frequente entre os nascimentos domiciliares do que naqueles ocorridos em hospitais; já a realização de menos de quatro consultas foi quase cinco vezes maior. Estudo de Silva et al.,15 que analisou dados da Região Metropolitana de São Paulo, identificou que fazer menos de 4 consultas foi 6,7 vezes mais frequente quando o parto ocorreu no domicílio comparado aos nascimentos hospitalares. A mesma pesquisa também observou que a o baixo número de consultas esteve associada aos óbitos neonatais precoces para as crianças que nasceram fora do ambiente hospitalar.

O pré-natal ausente ou o menor número de consultas associado ao parto acidental não-hospitalar foi um fator recorrente em diversos outros estudos.9,11,14,23 O efeito protetor da assistência prénatal se dá por meio da detecção precoce de condições desfavoráveis ao desenvolvimento da gestação e da intervenção sobre esses fatores. A atenção pré-natal oportuna é fundamental na identificação de gestações de risco e no encaminhamento para atenção hospitalar adequada ao parto e a eventuais necessidades de atenção intensiva ao recémnascido. Estudos mostram que mães em piores condições de vida e comportamento de risco na gravidez são as que mais ficam à margem da assistência pré-natal,21,24 e que existe relação entre a diminuição do número de consultas de pré-natal, conforme aumenta a vulnerabilidade à pobreza. 24
Esse estudo identificou risco de morte maior para os nascidos em domicílio ou outro local quando comparados aos nascimentos hospitalares, sendo quatro vezes maior para o componente neonatal precoce e 3,5 vezes para o pós-neonatal. Este resultado sugere que possíveis diferenças de acesso à atenção, no momento do parto, se mantem ao longo do primeiro do ano de vida desses recém-nascidos, pois não há relação direta entre nascer acidentalmente fora do hospital e a morte pós-neonatal; esta está mais relacionada a fatores determinados pelas condições de vida e características das famílias, entre as quais o nível educacional, renda, alimentação, condições da habitação e acesso a serviços de saneamento básico e de saúde. 20,21,25,26

Estudo realizado na Região Metropolitana de Belo Horizonte 26 identificou como fatores independentemente associados ao maior risco de óbito pósneonatal por diarreia e pneumonia: atraso vacinal, estado geral (grave) à admissão hospitalar, não realização de procedimentos hospitalares e desnutrição presente no momento da internação. Outro estudo identificou que a situação de iniquidade nas condições de vida exerce papel fundamental nos acentuados diferenciais de mortalidade pós-neonatal, por broncopneumonia e por doenças infecciosas intestinais no Recife. 25

$O$ fato de que, após ajuste pelas demais variáveis, não ter se encontrado efeito negativo dos nascimentos não hospitalares sobre a mortalidade neonatal precoce e tardia, mas apenas sobre os óbitos pós-neonatais, sugere que esse grupo era socialmente mais vulnerável ao nascer e que manteve sua vulnerabilidade ao longo do primeiro ano de vida. Como não há um efeito direto do local de nascimento sobre as causas de morte mais frequentes no período pós-neonatal, como por exemplo, as doenças diarreicas e pneumonia, esse resultado sugere que essas crianças podem estar mais expostas a condições de vida precárias, que estão associadas à ocorrência dos óbitos pós-neonatais. Considerando que a organização dos serviços de saúde privilegia os nascimentos em ambiente hospitalar, a ocorrência de nascimentos domiciliares acidentais pode ser considerada como um marcador de vulnerabilidade social. Esta hipótese é reforçada pelo fato que a baixa escolaridade materna também esteve associada ao aumento do risco de ocorrência de óbitos pósneonatais como confirmado em outros estudos. 27,28

A maior mortalidade associada aos partos fora do ambiente hospitalar condiz com os achados da literatura. ${ }^{9}$ Estudo de caso-controle em Glasgow encontrou associação estatística entre partos domiciliares acidentais e morbidade e mortalidade perinatal. $10 \mathrm{Na}$ 
Finlândia, após ajuste por peso ao nascer, a mortalidade perinatal foi quatro vezes maior para os nascimentos não hospitalares não planejados. ${ }^{16} \mathrm{Em}$ Pelotas, nascer fora da rede hospitalar também esteve associado com maior mortalidade neonatal. ${ }^{13}$ Estudo de revisão de McLelland et al. ${ }^{29}$ relata que os desfechos maternos e infantis são piores para os partos realizados antes de chegar ao hospital em relação aos que ocorrem nos hospitais, e que a situação de muitos desses recém-nascidos está comprometida pela prematuridade e baixo peso, levando frequentemente à necessidade de admissão em unidades de terapia intensiva e maior custo da atenção.

Observou-se maior mortalidade infantil para os neonatos com baixo peso ao nascer, confirmando que este fator se constitui em importante preditor do risco de morte. 20 No entanto, a probabilidade de morte entre os nascidos fora do hospital foi duas vezes maior nos dois grupos de peso analisados, o que indica que, independentemente do peso ao nascer, há um maior risco de óbito infantil para os nascimentos não hospitalares. Chama atenção o fato de que para os NV com peso igual ou superior a $2500 \mathrm{~g}$ essa probabilidade foi duas vezes maior para os nascimentos domiciliares/outro local. Em estudo realizado na Finlândia, após ajustes por peso do recém-nascido, idade materna, paridade e posição socioeconômica, a mortalidade perinatal dos nascidos com $2500 \mathrm{~g}$ e mais foi três vezes maior em relação aos que nasceram em hospitais. 16

Esta pesquisa mostrou que, no Estado de São

\section{Referências}

1. Christiaens W, Gouway A, Bracke P. Does a referral from home o hospital affect satisfaction with childbirth? A crossnational comparison. BMC Health Serv Res. 2007; 7: 1-9.

2. Martin JA, Hamilton BE, Sutton PD, Ventura SJ, Menacker F, Kirmeyer S, Munson ML. Births: final data for 2005. Natl Vital Stat Rep. 2007; 56 (6): 1-103.

3. Springer NP, Van Weel C. Home birth. BMJ. 1996; 313 : 1276-7.

4. Blais R. Are home births safe? Canadian Med Assoc J. 2002; 166: 335-6.

5. Janssen PA, Lee SK, Ryan EM, Etches DJ, Farquharson DF, Peacock D, Klein M. Outcomes of planned home births versus planned hospital births after regulation of midwifery in British Columbia. Canadian Med Assoc J. 2002; 166 : 315-23.

6. BEMFAM (Sociedade Civil Bem-Estar Familiar no Brasil). Pesquisa nacional sobre saúde materno-infantil e planejamento familial (PNSMIPF) Brasil, 1986. Rio de Janeiro; 1987.
Paulo, existe um segmento de partos acidentais ocorridos em domicílios e no trajeto para o serviço de saúde que merecem atenção, tanto por não serem atendidos em condições ideais como por registrarem maiores taxas de mortalidade infantil. Esses nascimentos reúnem maiores proporções de neonatos com condições biológicas mais precárias, como baixo peso ao nascer e gestações pré-termo, assim como mães em situações mais vulneráveis no que se refere à maior idade, baixa escolaridade e ausência de companheiro.

Do ponto de vista da organização da atenção à saúde materno-infantil, destaca-se o pré-natal ausente, o que é um alerta para as unidades de saúde. Tal situação pode ser entendida como evento sentinela para os serviços de saúde, indicando problemas de cobertura da atenção pré-natal e necessidade de treinamento dos profissionais para aprimorar a identificação, busca e o atendimento desse grupo de mulheres, bem como, após o parto, realizar o seguimento desses recém-nascidos, visando reduzir as mortes infantis evitáveis.

Há a necessidade de novos estudos para melhor compreender o contexto de vulnerabilidade dessas famílias, que ajudem a explicar o maior risco de morte pós-neonatal das crianças que nasceram acidentalmente fora do hospital.

\section{Agradecimentos}

Apoio financeiro da Fundação de Apoio à Pesquisa do Estado de São Paulo (Fapesp).

7. Ripsa (Rede Interagencial de Informações para a Saúde) Indicadores e Dados Básicos 2011. [acesso em $20 \mathrm{dez}$ 2013]. Disponível em <http://tabnet.datasus.gov.br/cgi/ tabcgi.exe?idb2011/f07.def>.

8. Brasil. Ministério da Saúde. PNDS 2006. Pesquisa nacional de demografia e saúde da criança e da mulher. Banco de dados. Brasília; 2008. Disponível em: http://bvsms.saude.gov.br/bvs/pnds/banco_dados.php

9. Viisainen K,Gissler M, Hartikainen AL, Hemminki E. Accidental out-of-hospital births in Finland: incidence and geographical distribution 1963-1995. Acta Obstet Gynecol Scand. 1999; 78: 372-8.

10. Rodie VA, Thomson AJ, Norman JE. Accidental out-ofhospital deliveries: an obstetric and neonatal case control study. Acta Obstet Gynecol Scand. 2002; 81: 50-2.

11. Almeida MF, Alencar GP, Novaes MHD, França Jr I, Siqueira AA, Schoeps D, Campbell O, Rodrigues LC. Partos domiciliares acidentais na região sul do Município de São Paulo. Rev Saúde Pública. 2005; 39: 366-75. 
12. McClure EM, Wright LL, Goldenberg RL, Goudar SS, Parida SN, Jehan I, Tshefu A, Chomba E, Althabe F, Garces A, Harris H, Derman RJ, Panigrahi P, Engmann C, Buekens P, Hambidge M, Carlo WA; NICHD FIRST BREATH Study Group. The global network: a prospective study of stillbirths in developing countries. Am J Obstet Gynecol. 2007; 197: 247e1-5.

13. Barros JD, Matijasevich A, Santos IA, Albernaz EP, Victora CG. Neonatal mortality: description and effect of hospital of birth after risk adjustment. Rev Saúde Pública. 2008; 42: 1-9.

14. Renesme L, Garlantézec R, Anouilh F, Bertschy F, Carpentier M, Sizun J. Accidental out-of-hospital deliveries: a case-control study. Acta Paediatr. 2013; 102: e1747.

15. Silva ZP, Almeida MF, Ortiz LP, Alencar GP, Alencar AP, Schoeps D, Minuci EG, Novaes HMD. Características dos nascidos vivos, das mães e mortalidade neonatal precoce na Região Metropolitana de São Paulo, Brasil. Cad Saúde Pública. 2009; 25: 1981-9.

16. Hemminki E, Heino A, Gissler M. Should births be centralized in higher level hospitals? Experiences from regionalized health care in Finland. BJOG. 2011; 118: 1186-95.

17. Waldvogel BC. Pesquisa pioneira recupera casos de AIDS no Estado de São Paulo: integração das bases do SINANAIDS e da Fundação Seade. Rev Bras Estud Popul. 2006; 23: $187-90$.

18. Fagerland MW, Hosmer DW, Bofin AM. Multinomial goodness-of-fit tests for logistic regression models. Stat Med. 2008; 27: 4238-53.

19. São Paulo (cidade). Secretaria Municipal da Saúde. Coordenação de Epidemiologia e Informação - CEInfo. O perfil dos nascimentos na cidade de São Paulo- Dez anos do SINASC. Boletim CEInfo Análise, ano VI, $\mathrm{n}^{\circ} 04$, Abril/2011. São Paulo; 2011.

20. Morais Neto OL, Barros MBA. Fatores de risco para mortalidade neonatal e pós neonatal na Região CentroOeste do Brasil: linkage entre bancos de dados de nascidos vivos e óbitos infantis. Cad Saúde Pública. 2000; 16 (2): 477-85.

Recebido em 6 de janeiro de 2014

Versão final apresentada em 14 de março de 2014

Aprovado em 2 de abril de 2014
21. Victora CG, Wagstaff A, Schellenberg JA, Gwatkin D, Claeson M, Habicht JP. Applying an equity lens to child health and mortality: more of the same is not enough. Child Survival IV. Lancet. 2003; 362 (19): 233-41.

22. Blodel B, Drewniak N, Pilkington H, Zeitlin J. Out-ofhospital births and the suply of maternity units in France. Health Place. 2011, 17: 1170-3.

23. Declerq E, MacDorman MF, Menacker F. Characteristics of planned and unplanned home births in 19 States. Obstet Gynecol. 2010; 116 (1): 93-9.

24. Minuci EG, Almeida MF Diferenciais intra-urbanos de peso ao nascer no município de São Paulo. Rev Saúde Pública. 2009, 43 (2): 256-66.

25. Guimarães MJB, Marques NM, Melo Filho DA, Szwarcwald CL. Condições de vida e mortalidade infantil: diferenciais intra-urbanos no Recife, Pernambuco, Brasil. Cad Saúde Pública. 2003; 19 (5): 1413-24.

26. Caldeira AP, França E, Goulart EMA. Mortalidade infantil pós-neonatal e qualidade da assistência médica: um estudo caso-controle. J Pediatr. 2001; 77 (6): 461-8.

27. Arntzen A, Mortensen L, Schnor O, Cnattingius S, Gisller $\mathrm{M}$, Andersen AMN. Neonatal and postneonatal mortality by maternal education - a population-based study of trends in the Nordic countries 1981-2000. Eur J Public Health. 2007; 18 (3): 245-51.

28. Barros FC, Matijasevich A, Requejo JH, Giugliani E, Maranhão AG, Monteiro CA, Barros AJD, Bustreo F, Merialdi M, Victora CG. Recent trends in maternal, newborn, and child health in Brazil: progress toward Millennium Development Goals 4 and 5. Am J Public Health. 2010; 100: 1877-89.

29. McLelland G, McKenna L, Archer F. No fixed place of birth: unplanned BBAs in Victoria, Australia. Midwifery. 2013; 29 (2): e19-25. 